\title{
DEVELOPMENT OF A WEB-BASED CUSTOMER-ORIENTED INTERACTIVE VIRTUAL ENVIRONMENT FOR MOBILE PHONE DESIGN
}

\author{
Peijun Wang ${ }^{a, b}$ \\ Robert Bjärnemo ${ }^{a}$ \\ Damien Motte ${ }^{a}$ \\ ${ }^{a}$ Lund University, Lund Institute of Technology \\ Division of Machine Design, Department of Design Sciences \\ P.O. Box 118 \\ Lund, SE 22100 \\ Sweden \\ Tel: +46 46 2228510, Fax: +46 46 2228060, Email: peijung.wang@mkon.Ith.se \\ bouthwest Jiaotong University, Department of Mechanical Engineering \\ Chengdu, 610031 \\ China \\ Email: wangpi@hotmail.com
}

\begin{abstract}
During recent years, mobile phone companies have experienced market-related difficulties that result in increasing competition globally. In an attempt to maintain or increase their market share, enterprises reorganize their resources and employ new technologies to sharpen their competitive edges. Moreover, with the globalization, more and more companies become multi-sited. Efficient and timely collaboration and communication among the development team members become critical for product success. This paper proposes a web-based interactive virtual environment for mobile phone design, named VMPDS (Virtual Mobile Phone Design Space). The features of the system include the combination of web technology and VR (Virtual Reality) technology, the ability to deal with and extend the product model database, adequate interaction for 3-D product model modification and customization, and usefulness for the examination and evaluation of product concept design. The system is implemented by using standard open technologies such as VRML (Virtual Reality Modeling Language), Java, SAI (Script Authoring Interface) and EAI (External Authoring Interface) to assure accessibility and platformindependence.
\end{abstract}

Keywords: Virtual reality; VRML; Concept design; Mobile phone.

\section{INTRODUCTION}

Mobile phone companies are facing a rapidly-expanding global scale market. For example, the average annual growth rate for China's wireless market has exceeded 100 percent over the last decade [1]. There are clear indications that the market will improve and continue to grow. In the meantime, the competition among companies is becoming much fiercer than ever before. The increasing competitiveness on the global market is forcing companies to adopt new technologies and reorganize their resources to promote productivity, including upgrading product quality, cutting costs, improving after-sell services, reducing time-to-market and investing a significant amount of their budget in marketing. Moreover, in order to stay competitive and profitable, many enterprises are becoming multi-sited, operating with partners and customers on a global market. However, in spite of these efforts, insignificant attention is being paid to identifying customer needs. The fact is that the increased competition is driving manufacturing away from mass production, where the manufacturer tells the customer what to buy, to mass customization, where the customer 
decides what the manufacturer will make [2]. In this case, effective collaboration and communication among designers and customers play a crucial role for the success of mobile phone companies.

Many factors influence the success of mobile phone products, such as technological advance, product price, attractive design, rich functionality, marketing, etc. Among them, customer preference is one of the most important factors. As a result, collecting and identifying customer needs and understanding their preferences are essential tasks in the development of new mobile phone products. However, it is demanding and challenging for designers to transfer customer-implicit preferences into product design specifications. One reason is that customer requirements and value preferences vary greatly from person to person because they have different backgrounds, including culture, tradition, personality, education and nationality, etc. Ming C. Chuang et al. investigated perceptual factors underlying user preferences about the product form of mobile phones [3]. There were "one hundred and two users who participated in a semantic differential test and graded the mobile phone samples according to their first impression of the specific image word and preference"[3]. But this test was constrained in the following ways: the first was that the product samples were described in a text and 2-D image formats, which could not depict the product fully. The second was that the number of interviews was limited due to the time factor. The last was that participants only graded the given mobile phone samples without the possibility of submitting their own implicit preferences. It is believed that web-based conceptual design techniques can improve efficiency by first building conceptual design models to represent products' geometries, structures, etc. and then distributing the models over the web for remote evaluation and verification of the design correctness [4]. In this paper we propose a web-based interactive 3-D virtual mobile phone design environment, called VMPDS (Virtual Mobile Phone Design Space). Our goal is to allow collaboration, communication, modification and evaluation to take place in the earlier, more creative phase of the mobile phone design process. VMPDS is a collaborative system that supports teamwork in different places and different times. It is mainly based on the technologies of VRML, Java, SAI (Script Authoring Interface), EAI (External Authoring Interface) and the Internet. The features of the system consist of 1) the combination of web technology and 3-D visualization technology; 2) the user-friendly and intuitive 3-D interactive user interface; 3) system scalability and accessibility and 4) minimal requirements on bandwidth and hardware. There have been some applications using VRML, Java and EAI [5, 6 , 7]. Dace A. Campbell set up a prototype used to disseminate construction design data as VRML models and HTML text to the design client and contractor [8]. Vincent G. Duffy et al. presented an Internet-based virtual reality facility layout system that can be used to test in industry as well as in the laboratory to help improve workplace safety [9]. However, most of these applications were real-time, walkthrough simulations and key-frame animations in which users can manipulate the 3-D model, control animation behaviors and change model attributes, such as color, size and scale, etc. In addition, only simple geometry and example models are treated, rather than complex and real industrial products. Markus Durstewitz et al. presented a virtual collaboration environment for aircraft design [7]. It focused mainly on collaborative review of the shared object and communication facilities. Its editing functions included changing color, size and position, or making the part invisible. Compared to these existing systems, the main innovations of VMPDS are: 1) the product is accurately modeled using CAD software, not just a simple, rough or conceptual representation; 2) this system has scalability due to the combination with a product database; 3) the dynamic I/O operation on VRML scenes is achieved and 4) VMPDS is a complete system, not only a 3D visualization. In VMPDS, users are involved in the product design activities and can submit their preferences on mobile phone style to the database that will serve as the product design benchmark to help designers adopt a proper design for the intended end users. With VMPDS, we can change traditional and costly design-manufacture-test-modify to design-simulate-refine-manufacture that is far more efficient from a resource point of view. We accomplish the network communication by a client/server structure using HTTP protocol (Hypertext Transfer Protocol). A Java-enabled web browser together with a VRML plug-in is the only requirement for the execution of VMPDS on an ordinary desktop PC.

The organization of this paper is as follows. Section 2 introduces the main tools used for developing VMPDS. Section 3 gives an overview of the VMPDS system architecture. The implementation of the user interface is depicted in details in Section 4. Section 5 describes the exchange and optimization of 3-D model data. We outline the network and database structure in Section 6. Section 7 discusses some weak points of VRML that we encountered in the development of VMPDS. Finally, Section 8 ends with our conclusions and proposals for future work.

\section{SELECTING VMPDS DEVELOPMENT TOOLS}

As described above, VMPDS is based on the integration of VRML, Java, SAI, EAI and the Internet. We intend to provide an interactive visual environment using VR that simplifies the collaboration and communication among designers and the user group located in geographically remote sites. One important criterion that influences the choice of VR tools is that we want this application to use as low bandwidth and hardware sources as possible in order to be accessible even through dial-up manner. To achieve this goal, three open standards are employed. We introduce them in the following order.

\subsection{VRML}

We use VRML to create 3-D virtual environment. VRML is a web-based technology for specifying 3-D interactive visualization over the Internet via a plug-in. There have been a number of studies targeting web applications using VRML (e.g., [10,11,12]). Compared to other 3-D visual VR development software, VRML has the following advantages: 
- VRML is non-proprietary and platformindependent. It is written in ASCII-based characters, and a plain text processor can edit it without the need of special licensing authoring tools. Moreover, most VRML browsers are freely available;

- VRML can be extended through such means as adding Java code or JavaScript into its Script nodes. Java has complete programming capabilities plus network access. The conjunction of VRML and Java through the Script node makes it possible to define more complex animations [13]. Also, like HTML, it can contain inlined and anchored URLs to link to outside worlds;

- Its latest version, VRML97 [14], was accepted as an ISO standard (ISO/IEC14772). Although tens of 3D file formats are available, none of them is successfully playing a role as an industry standard [15]. VRML is the first open international standard of its kind [15]. Apart from VRML, there are many 3-D visualization softwares, such as Cult3D ${ }^{\circledR}$ (Cycore Corporation), MultigenCreator ${ }^{\circledR}$ (MultigenParadigm Inc.), AtmoSphere ${ }^{\mathrm{TM}}$ (Adobe Systems Int.), WorldUp ${ }^{\circledR}$ (Sense $8^{\circledR}$ Corporation), MockUp ${ }^{\circledR}$ (Parametric Technology Corporation), 3DS Max ${ }^{\circledR}$ (Autodesk Int.) and Maya ${ }^{\circledR}$ (Alias|Wavefront ${ }^{\mathrm{TM}}$ ), etc. Although many of them have higher rendering quality than VRML, the disadvantages are not negligible. First, in contrast to VRML, most of them are proprietary and platform-dependent, which limits their wide-scale availability. Second, some of them fail to enable concurrent access and therefore are inadequate for a real-time, web-based collaboration application. Next, some of them lack the capability of integrating programming language to enhance interactivity. Last, but not least important, most of their resulting scene files are too large to be handled in the Internet;

- A VRML file is very compact. Furthermore, some compression approaches can reduce the VRML file size significantly. For example, an approach to compressing large VRML scene files was introduced in [16] that achieved compression ratios of $50: 1$ or more. We pay great attention to the 3-D scene file size because the existing Internet service in many developing countries where big markets exist and mobile phone companies are establishing their manufacturing factories offers limited bandwidth. Even in some developed countries like Sweden, where broadband is very common, some people still get into the Internet via dial-up methods. So, VMPDS demands as small 3-D scene files as possible. VRML can meet this demand.

\subsection{Java and JavaScript}

As described above, VRML has the capability of using Java and JavaScript in its Script node. It is well known that Java has strong network capability, with promising distribution mechanisms that make web-based application easier. In VMPDS, both Java and JavaScript are used in the URL fields of Script nodes. JDK (Java Development Kit) 1.2 [17] is used to compile Java files into byte-code Class files that will be interpreted by JVM (Java Virtual Machine).

\subsection{SAI and EAI}

Two approaches are used to connect VRML files with external Java programs. One is SAI, which uses JavaScript to communicate with Script nodes of VRML files. Another is EAI, which is an application interface for Java [18,19]. We use EAI as a channel to connect the VRML world with HTML pages through Java applets that control the VRML scene dynamically. Figure 1 shows the communications between VRML files and Java codes.

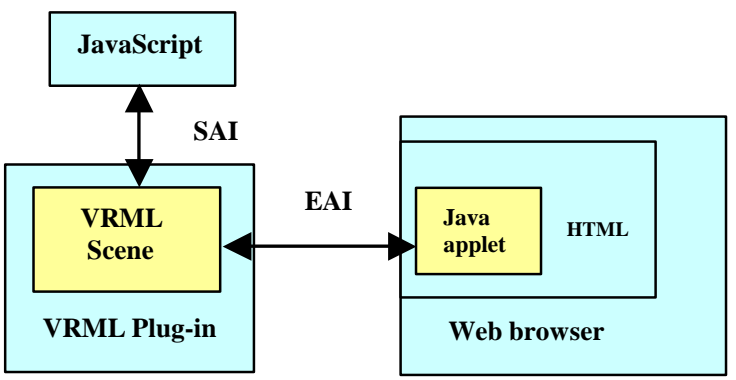

Figure1. Communication channels

\section{SYSTEM ARCHITECTURE}

VMPDS is intended to provide a compact, low hardware demand and customization environment to facilitate webbased collaborative design and evaluation tests. Without using special hardware such as high performance graphic acceleration cards, VMPDS allows any user to enter VMPDS by visiting its homepage, shown in Fig. 2.

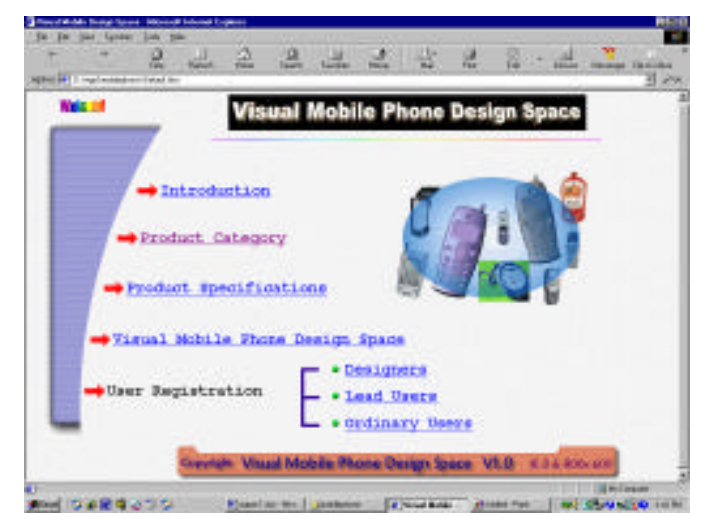

Figure 2. A snapshot of the homepage of VMPDS

As shown in Fig. 3, there are four subsystems in VMPDS: User Registration, Virtual Mobile Phone Design Space, Product Category and Product Specification.

The virtual design space is divided into three parts. The first is the 3-D GUI (Graphic User Interface) through which 
most of the interactive design and modification are performed; the second is the 3-D mobile phone product visualization environment where users can manipulate and observe the product model from any desired perspective; the last part provides network support and connection to the model database. Figure 4 depicts the architecture of the virtual design space.

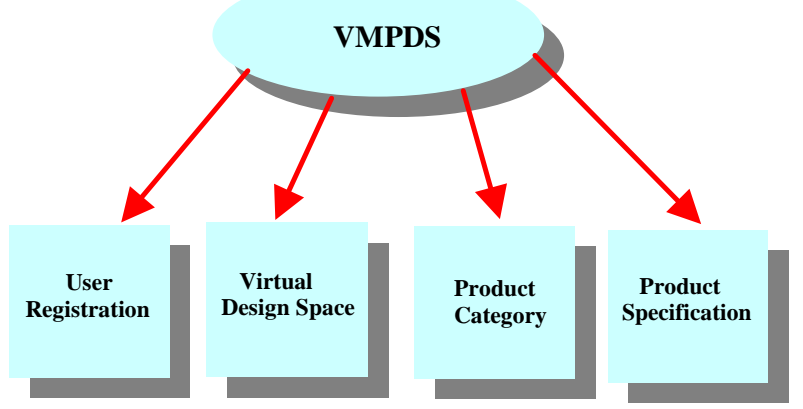

Figure 3. The organization of VMPDS

\section{DEVELOPMENT OF USER INTERFACE}

In order to be accessible for various users, some of whom have no significant computer expertise, an intuitive user interface is proposed in VMPDS. The window is split into three parts. On the left is the control panel used for basic design, modification and customization. The middle part, occupying most of the screen, is the 3-D mobile phone model. On the right are four groups of fine-control sliders used for quantitative modification operation. The I/O operations are performed through the Java Applet buttons named "Clear", "Save", "Import" and "Evaluating", etc. which are on the same html page as the 3D VRML scene. As a virtual reality application, VMPDS permits a virtual phone call by clicking the digital keys and functional keys, respectively. The LCD screen of the mobile phone model will display the input content together with sound response. This is fulfilled through attaching Touch Sensor nodes to every key in the VRML world and using JavaScript coding in the Script node. Figure 5 shows a snapshot of the user interface.

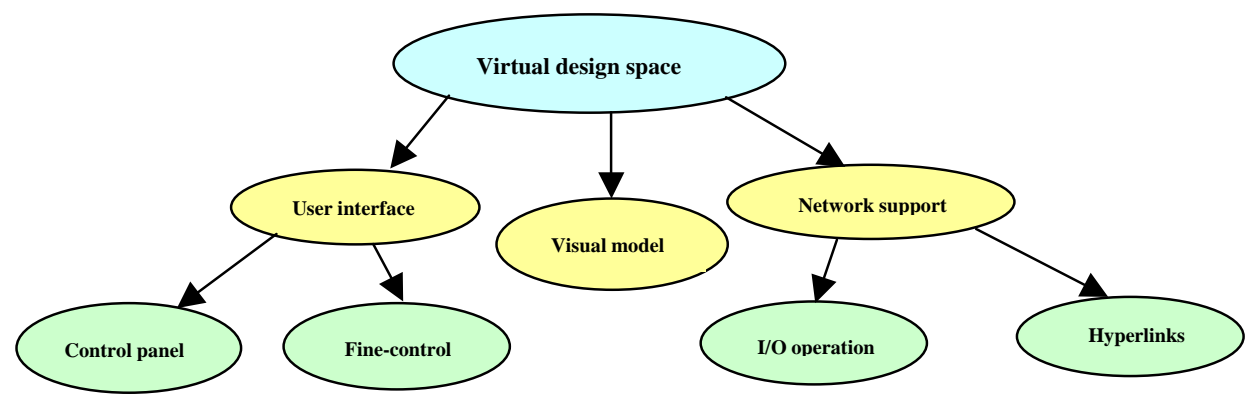

Figure 4. The architecture of the visual design space

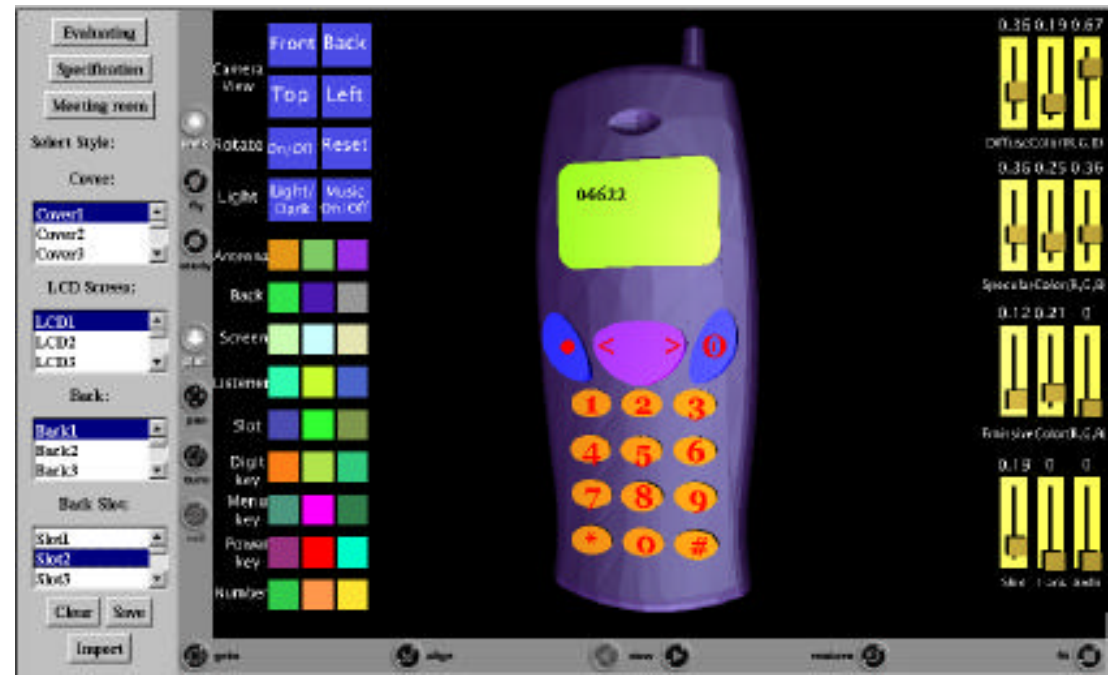

Figure 5. A snapshot of the user interface in VMPDS 


\subsection{Function analysis of control panel}

The control panel, on the left of the screen, consists of two parts, giving the user access to the full functionality of the system. One is immersed in the VRML scene and accesses collection of buttons. Another is Java Applet buttons and pull-down lists, as shown in Fig. 5. We divide mobile phone products into a few basic classes according to their essential appearance characteristics. With regard to a certain class, users can choose various styles through selecting items from the pull-down lists to get different combinations of the cover, the LCD screen, the back and the back slot. The basic functionality of the control panel includes:

- Viewing from among pre-defined viewpoints (Figure 6 is a snapshot of the Left viewpoint of the model). Vincent G. Duffy et al. described in [9] that even an expert in VR cannot accurately arrive at a specific point consistently. Although the VRML plug-in has similar functions to change the position and rotation of the $3 \mathrm{D}$ object, it is difficult to position the model exactly in a certain orientation, such as Top, Front, Left and Back, which are very common perspectives in CAD domain;

- Playing rotation animation or stopping animation at any time;

- Resetting the scene to the default state and eliminating all the modifications;

- Switching the product styles (Figure 7 shows a snapshot of switching to another style when the Cover3, LCD3, Back1 and Slot2 are selected);

- Selecting colors of mobile phone components;

- Customizing 3-D design environment, such as lighting, background music, etc.

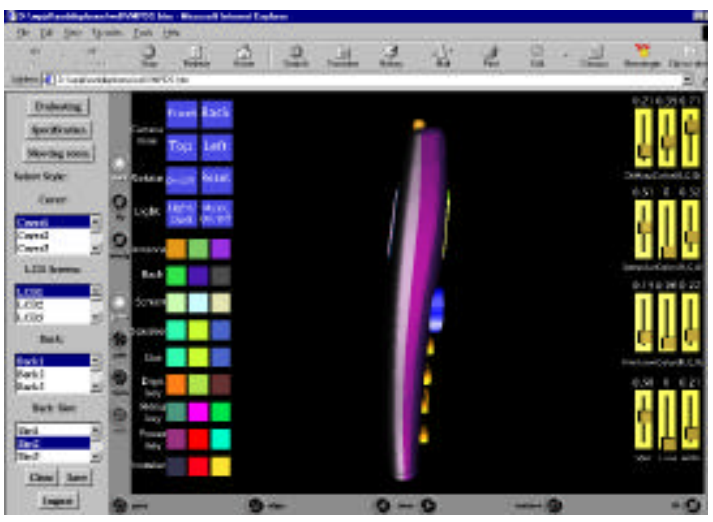

Figure 6. A snapshot of Left viewpoint of the model

Inside the VRML scene, all the buttons on the control panel are arranged using HUD (Heads Up Display) technology. The control panel always follows the user around no matter how the 3-D scene is rotated or moved. This means that all the buttons are kept in their initial positions facing the user. We achieve this requirement by using a sufficiently large Proximity Sensor in the VRML world.

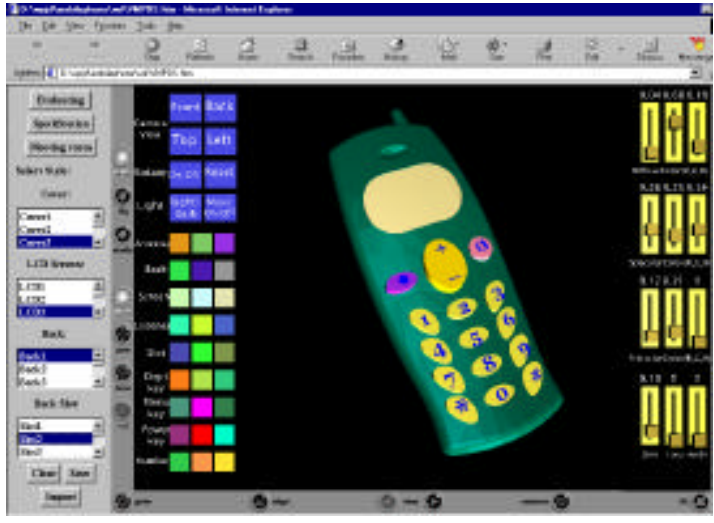

Figure 7. A snapshot of switching to another style

\subsection{Mobile phone model visualization}

In order to make effective interactions and modifications, the mobile phone model consists of ten parts: upper body (cover), back, digital keys (from "0" to "9"), functional keys (including “\#”, “*”, etc.), antenna, battery, brand slot, LCD display screen, text and lid. All of these parts can be modified individually, corresponding to user input. Whenever loaded into the 3-D environment, the model is always set to the default state that is carefully selected so as to give the user a proper first view. The user can navigate in the 3-D scene, move around, rotate and zoom the model freely. Moreover, VMPDS allows the user to detach and assemble the model by using the mouse to drag the cover. Figure 8 shows the upper body detached from the model body. In this way the user can have a close look at the element and the interior structure separately.

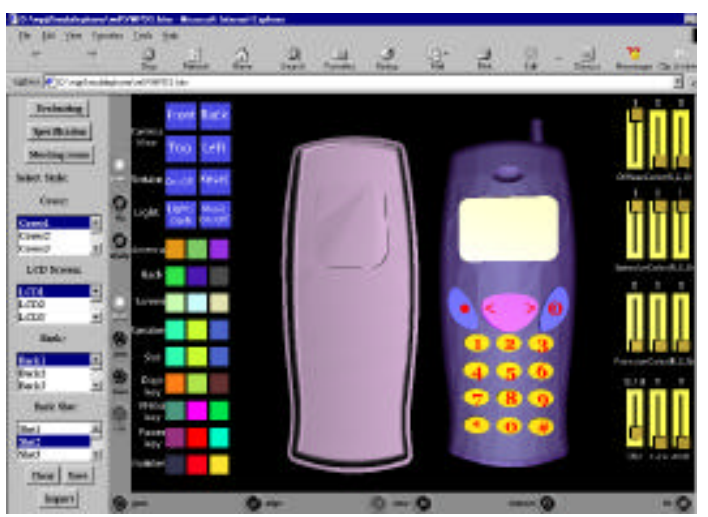

Figure 8. A snapshot of the detaching operation

\subsection{Fine-control sliders}

For every mobile phone style, users can customize their preferred appearance attributes quantitatively using four groups of control sliders on the right of the screen. By dragging the slider up and down, the diffuse, specular and emissive color can be changed. Furthermore, the user can play with the transparence, shininess and ambient intensity to customize a desirable mobile phone. Every modification is displayed at the top of the corresponding slider in the form of digits ranging from 0 to 1 . Fine-control sliders provide users 
with more optimization capability in an intuitive and quantitative way.

\subsection{Input/Output operation}

VMPDS features extensibility and scalability. The following three operations are designed for this purpose. After clicking the button "Clear", the user removes the whole model from the scene. The button "Import" is linked to a HTML interface that is embedded with a Java applet connected to the model database. In section 5 we describe the approach to creating, converting and storing the CAD data in the product library.

Furthermore, the user can save the customized model by clicking the button "Save" that is also linked to the HTML interface. Of course, only the designers and selected users have permission to work with the database. We divide VMPDS users into three groups; one consists of designers, marketing personnel and product managers, etc. Another group is called lead users, who are carefully selected and given passwords for access to the database. The last group is general users who can manipulate the model in 3-D scene and submit their evaluating comments. Figure 9 shows a snapshot of the user registration system. This system is used not only for security checks but also for collecting user information.

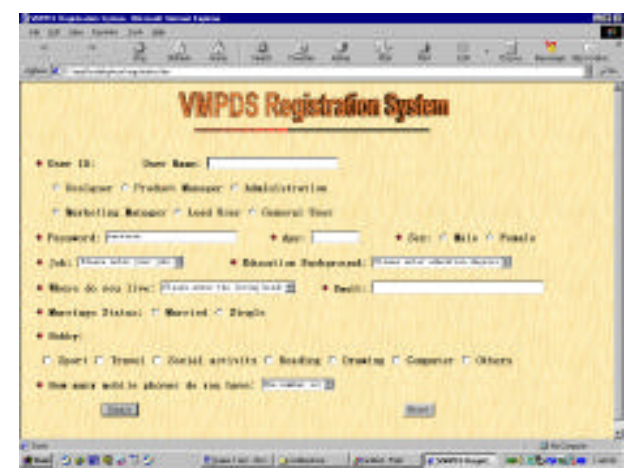

Figure 9. A snapshot of the registration system

\subsection{Hyperlinks}

VMPDS is developed not only for interactive design but also for analyzing, evaluating and communicating. On the upper left corner of the screen are three buttons marked "Evaluating", "Specification" and "Meeting room" respectively. Users can submit their evaluations by clicking the button "Evaluating" and jump to the HTML form where they input their comments and rate the product's conceptual design. After some analyzing and statistical processing, the company can have a good insight into customer preferences. Moreover, by clicking the button "Specification", the user will access the production specification where production introduction, possible price, component materials and weights, size, technological features as well as the website of the company are given in the forms of text and 2-D image. Finally, we intend to provide an interactive tool for real time video information through "Meeting room" to aid the communication between designers and users. This part of the work is underway.

\section{EXCHANGE AND OPTIMIZATION OF 3-D MODEL DATA}

There are two main methods for creating VRML 3-D models. One is to directly use the geometry nodes defined inside VRML, such as Box, Cone, Cylinder, Sphere, IndexedFaceSet, ElevationGrid and Extrusion, etc. The resultant file is very compact. But it is difficult and, in some cases impossible, to create realistic and complex models with only these primitive nodes. In the development of VMPDS the second method is used. The mobile phone model is created accurately using CAD software packages, then the CAD data is exported to VRML format. Currently many CAD packages have this exporting capability, among them are Pro/Engineer (Parametric Technology Corporation), UGII (Electronic Data Systems Corporation), SolidEdge (Electronic Data Systems Corporation), SolidWorks $®$ (SolidCompany), AutoCAD (Autodesk, Inc.) and CATIA ${ }^{\mathrm{TM}}$ [20], etc. Designers and users can create their own model by using any kind of CAD software. We predefine three datum planes and alignment axes as well as size units. The back part of the mobile phone is selected as the base part, and other elements are integrated into this base. Following these rules will make new components fit the VMPDS and achieve easy assembly. Considering the diversity of user software platforms, it is necessary to store the 3-D model data both in VR format and other compatible and neutral CAD formats to ensure quick, easy, reliable and flexible sharing and exchanging of product data information among design teams, possibly distributed all over the world.

There are no standard methods for the data translation from CAD into VR. In our case, the models are built using Pro/Engineer and then exported to 3DS Max, where they are edited and optimized further in the formats of IGES (Initial Graphics Exchange Specification) and STEP (Standard for Exchange of Product model data), both of which are nonproprietary standards and usable widely for CAD data exchange.

The converted VRML file from CAD software packages tends to be very big. The main reason is that VRML uses polygons instead of non-linear free-formed surfaces, such as NURBS (Non Uniform Rational B-Spline) to describe 3-D objects. The more accurate the model, the more polygons have to be used. As a result, a compromise between realistic fidelity and rendering speed has to be made. Since the mobile phone is a consumer product used daily, attractive appearance is crucial for the success of product design. Thus a high quality 3-D model is necessary in VMPDS. On the other hand, VMPDS aims at being available on ordinary user desktop PCs through the Internet without using special high performance hardware. In this case, we optimized the 3-D model data through the following methods:

- Using some software, like 3DS Max, to reduce the polygon number;

- Simplifying less important parts; 
- Using as few texture maps as possible. We assign fundamental colors on most of the elements and employ texture maps in very few components, such as "brand slot";

- Using PROTO nodes in the VRML world. Geometry, property, behavior and even animation can be encapsulated and reused through prototyping;

- Compressing the VRML file. There are many effective compression tools available such as GZIP. The VRML browser can render the compressed VRML scene without apparent time delay;

- Using LOD (Level of Detail) technology. Different degrees of display details for the same component are defined. At any given time, only one of them is displayed according to the distance between the viewer and the object. The closer the viewer gets to the object, the higher the level of detail displays. Low detailed objects contain few polygons. As a result, we can reduce the total polygons of the 3-D scene. But LOD introduces a new problem: it takes more download time. Also, too few details cause less realistic and unattractive results. We have to make a trade-off between rendering and downloading.

It should be noted that, from our experience in the development of VMPDS, a STEP file gives a better converting result than IGES format. Some geometric surface information was lost when we imported IGES files to 3DS Max, while STEP files can maintain almost all geometry features among different CAD package platforms.

The possibility of changing the individual geometric shapes dynamically and quantitatively, from both VRML interface and HTML interface through Java Applet, depends on the complexity of the object. For simple geometries, like box, cone, sphere, etc. and their combinations, the interactive modification is easy. For geometries that can be described using some mathematical equations and other approaches, the quantitative changing is also possible. Figure 10 explains the quantitative modification process.

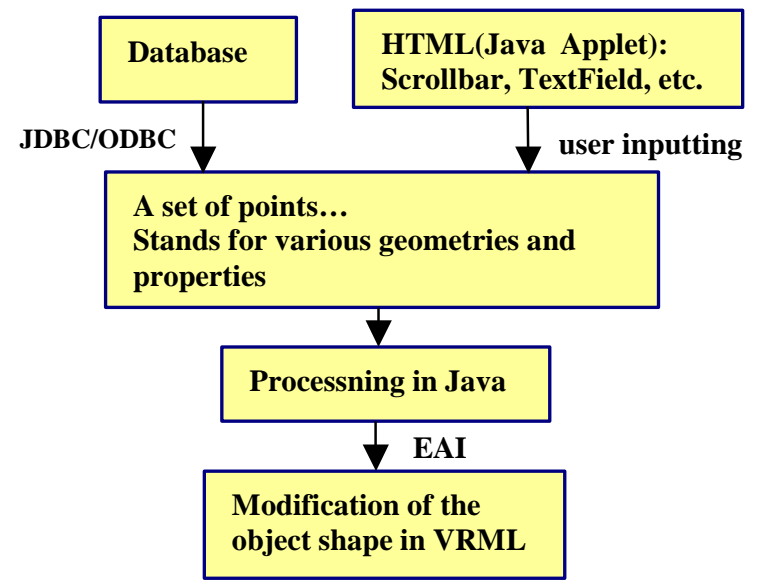

Figure 10. Quantitative modification process
But for complex geometries that are converted from CAD software, the quantitative modification is difficult and time consuming. The first reason is that the converted object in VRML consists of huge numbers of points inside a set of IndexedFaceSet nodes and the spatial logical relations among these points are non-linear. Another reason is that, as shown in Fig. 11, it is difficult to find the match between the point on the $3 \mathrm{D}$ model and its corresponding point in the VRML file.

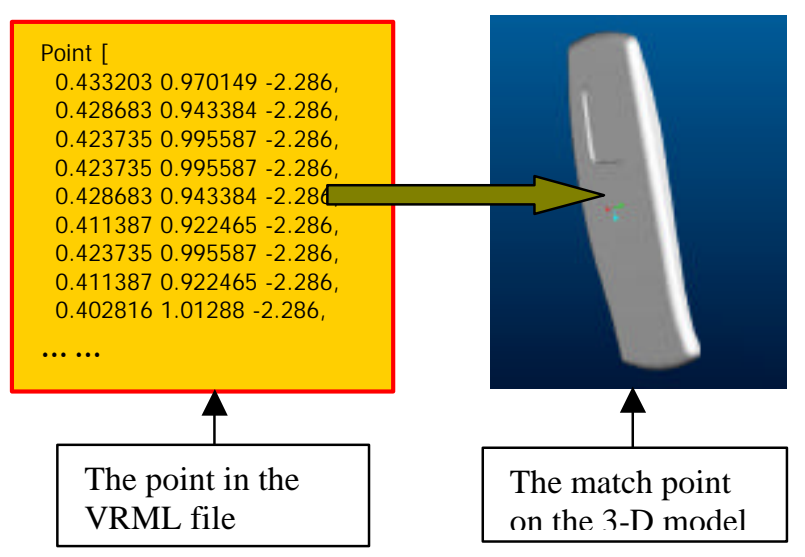

Figure 11. Match relation between the points

As a result, in VMPDS every basic element, such as the back, the LCD display screen and the upper body, etc. converted from ProE CAD software, is considered as the minimum unit for modification.

\section{NET-WORKING AND DATABASE STRUCTURE}

In VMPDS, a client /server architecture and the standard HTTP protocol are adopted, allowing multiple networked designers and users to work in the same visual space. Figure 12 describes the network structure. Although such a structure could be a bottleneck for networks with low bandwidth, we intend to use this solution because it is a comparatively simple approach. Another reason is that the whole size of VMPDS is controlled as little as possible.

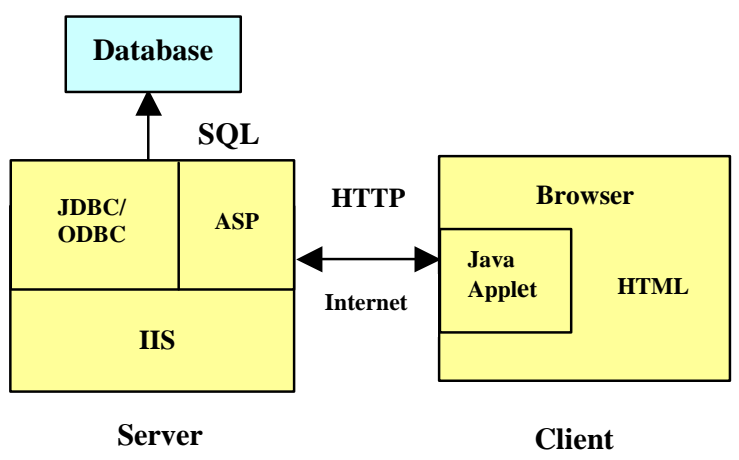

Figure 12. Network and database structure 
The client initiates the communication by sending a message to the server through a HTML file that is embedded with Java applets. Afterwards the VRML file is downloaded into the client browser and rendered locally. As shown in Fig. 10, the database is updated and queried using SQL (Structured Query Language) commands through an open database connection (ODBC). The communication between the database and HTML files on the client side is fulfilled through ASP (Active Server Pages). HTML files are used for generating the forms that collect the input/output operation from the user and sending them to the ASP script on the server. We use ASP instead of CGI (Common Gateway Interface) because ASP is simpler and easier to edit due to its ASCII script feature. Moreover, it has higher efficiency than CGI. On the server side, Microsoft's Internet Information Server (IIS) is used. Through the combination of ADO (ActiveX Data Object) and ASP, VMPDS enables users to add or remove the model file from the HTML file directly.

Two product libraries exist in VMPDS. One is the basic product library maintained by designers and accessible only for designers and lead users. Another is the public product library that is a subset of the basic product library and accessible for any user. The second library stores the mobile phone models intended to be evaluated and customized by customers. Figure 13 gives a snapshot of the mobile phone product category that is the public product library. In the product category, models are arranged according to their specialties. Following is one of the prototype specialties:

- Asymmetrical body;

- Large and rounded rectangle LCD display screen;

- No lid;

- Elliptical digital keys arranged symmetrically;

- Sharp color contrast between the upper body and the back.

At present, the database is in a prototype version and is under construction.

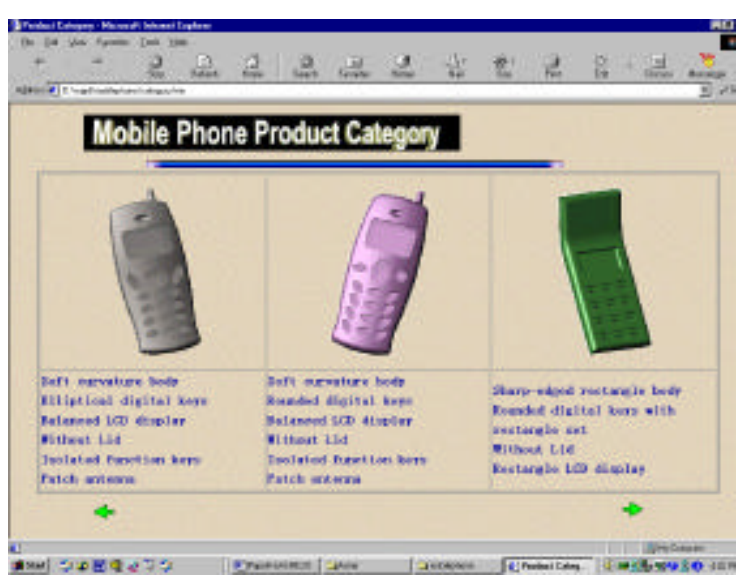

Figure 13. A snapshot of the product category

\section{DISCUSSION OF THE DEFICITS OF VRML}

Although VRML technology is superior to many other 3-D simulation tools of its kind and fairly robust for visualization, it is still relatively immature and insufficiently defined for complex interaction, big 3-D scene creating and rendering. In this section we discuss its weak points that we encountered while developing VMPDS.

- The VRML standard is not fully implemented by some VRML plug-ins. Different plug-ins render the same scene differently. In other words, the compatibility is not very satisfactory. For example, in our case, WorldView2.0 does not support the mid format of sound and does not fully support EAI;

- The VRML standard itself, to some extent, is not well defined. For example, as VRML itself lacks the ability to deal with a filestream, such as I/O, the interactively-renewed scene files have to be saved by storing the changed node information in a database or a specially-defined file through Java;

- JavaScript cannot manipulate nodes inside the inlined nodes;

- Collision can only be detected between the viewer and objects, but not between objects themselves. This causes some problems in VMPDS: when an element of a mobile phone model is dragged away from the model body, it is possible for it to "go through" into other elements. This does not happen in physical reality;

- VRML 97 does not provide non-graphical attributes of objects, like gravity, friction and elasticity, etc. that are necessary to create a virtual environment. Besides, there is no Boolean operation in VRML. As a result, it is difficult to model complex objects by directly using VRML;

- Text in other languages, such as Chinese, Japanese and Korean, etc. is not well supported by plug-ins;

- Lighting effects are not perfect. VRML objects do not cast shadows or reflect light;

- Compared to some commercial 3-D visualization software, like 3DS Max and Maya, VRML authoring tools are far from being sophisticated;

- No keyboard input capability.

We expect that the next generation of VRML, which will bring XML, MPEG4 and VRML closer, could overcome these drawbacks.

\section{CONCLUSIONS AND FUTURE WORK}

This paper presents a web-based interactive visual mobile phone design system called VMPDS in detail. VMPDS provides intuitive and effective means for designers and unsophisticated users, who could be distributed at geographically remote places, to manipulate, modify, evaluate, comment on mobile phone product conceptual designs and communicate with each other. VMPDS can be applied in the earlier and more creative phase of the design process rather than a walkthrough of final design, offering a potential for large cost savings. Moreover, using VMPDS to 
identify customer needs has obvious advantages over traditional interviews or other media. With VMPDS, companies can have more information to go on when developing new products, and the design team can get optimal design solutions from a large number of usercustomized alternatives. Furthermore, VMPDS will facilitate Design on Demand (DoD). We believe that in the future customers could order their own mobile phones through such a system as VMPDS, just like the practice in the automobile industry today.

Our research work proves that VRML, Java, SAI, EAI and ASP are, to some extent, sufficiently powerful to develop a web-based collaborative design environment, although there are some shortcomings in VRML that are also discussed in this paper. The development approach proposed in this paper provides some useful experience for designing a web-based 3-D interactive visual application. The techniques described here can be applied to other web-based visual product design.

VMPDS is implemented and tested on Windows NT4.0, Windows XP and Windows 98 using Internet Explorer 6.0 and Internet Explorer 5.5 as web browsers. The VRML plugins used are Cortona 4.0 (Parallel Graphics Ltd.) and CosmoPlayer 2.0 (Silicon Graphics Inc.).

VMPDS has not been fully developed. Further development work is underway, including a complete integration of a server-based database with the 3-D scene and the development of the visual meeting room that will provide designers and users with an efficient and convenient communicating tool for transferring verbal information. In addition, the mobile phone model library needs to be further enriched.

\section{REFERENCES}

[1] George, L., 2001, "China seeks CDMA," Electronic Engineering Times, pp. 38-38.

[2] Kuljis, J., Paul, R. J., 2000, "A Review of Web Based Simulation: Whither We Wander?" Proceedings of the 2000 Winter Simulation Conference, Vol.2, pp. 18721881.

[3] Chuang, M. C., Chang, C. C., Hsu, S. H., 2001, "Perceptual factors underlying user preferences toward product form of mobile phones," Industrial Ergonomics, 27, pp. 247-258.

[4] Qin, S. F., Harrison, R., West, A. A., Jordanov, I. N., Wright, D.K., 2003, "A framework of web-based conceptual design," Computers in Industry, 50, pp. 153164.

[5] Flake, S., Geiger, C., Mueller, W., Ruf, J., Paelke, V., Rosenbach, W., 2001, "Customer-Oriented Systems Design through Virtual Prototypes," Proceedings of Tenth IEEE International Workshops, pp. 263-268.

[6] Dos Santos, C.L.N., Cunha, G. G., Landau, L., 2001, "Use of VRML in Collaborative Simulations for the Petroleum Industry," Proceedings of 34th Annual of Simulation Symposium, pp. 319-324.

[7] Durstewitz, M., Kiefner, B., Kueke, R., Putkonen, H., Repo, P., Tuikka, T., 2002, "Virtual Collaboration Environment for Aircraft Design," Proceedings of the
Sixth International Conference on Information Visualisation, pp. 502-507.

[8] Campbell, D. A., 2000, "Architectural construction documents on the web: VRML as a case study," Automation in Construction, 9, pp. 129-138.

[9] Duffy, V. G., Wu, F. F., Ng, P. P. W., 2003, "Development of an Internet virtual layout system for improving workplace safety," Computers in Industry, 50, pp. 207-230.

[10] Rojdestvenski, I., Cottam, M., 2002, "Visualizing metabolic networks in VRML," Proceedings of the Sixth International Conference on Information Visualisation, pp. 175-180.

[11] Ramos, J. J.G., Maeta, S.M., Bergerman, M., Bueno, S.S., Mirisola, L.G.B., Bruciapaglia, A., 1999, "Development of a VRML/Java Unmanned Airship Simulating Environment," Proceedings of the IEEE/RSJ International Conference on Intelligent Robots and Systems, Vol.3, pp. 1354-1359.

[12] Buffa, M., Lafon, J.-C., 2000, "3D virtual warehouse on the WEB," Proceedings of IEEE International Conference on Information Visualization, pp. 479-484.

[13] Tamiosso, F. S., Raposo, A. B., Magalhaes, L.P., Ricarte, I.L.M., 1997, "Building Interactive Animations Using VRML and Java," Proceedings of IEEE Computer Graphics and Image, pp. 42-48.

[14] VRML Consortium. The Virtual Reality Modeling Language: International Standard ISO/IEC DIS 14772-1, 1997. Also available at http://www.vrml.org/Specifications/VRML97

[15] Jang, E. S., 2000, "3D Animation Coding: its history and framework," IEEE International Conference on Multimedia and Expo, Vol.2, pp. 1119-1122.

[16] Taubin, G., Horn, W. P., Lazarus, F., Rossignac, J., 1998, "Geometry Coding and VRML," Proceedings of the IEEE. Vol.86, pp. 1228-1243.

[17] Java Language Documentation, http://java.sun.com

[18] Silicon Graphics, Inc. The External Authoring Interface(EAI), http://cosmosoftware.com/developer/eai.html

[19] Marrin, C., External Authoring Interface. http://vrml.sgi.com/movingworlds/spec/Externalinterfac e.html

[20] http://Www-1.ibm.com/industries/plm 